\title{
Isometric Mapping Hashing
}

Yanzhen Liu, Xiao Bai, Haichuan Yang, Zhou Jun, and Zhihong Zhang

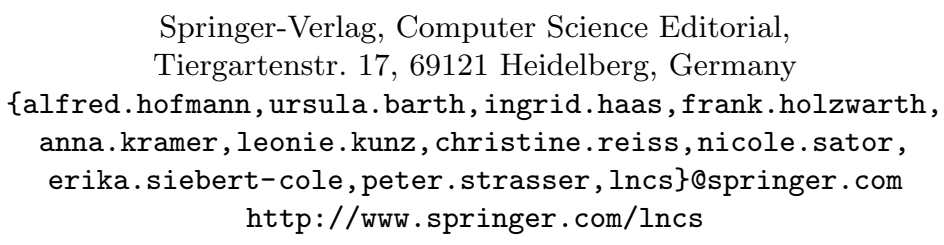

\begin{abstract}
Hashing is a popular solution to Approximate Nearest Neighbor (ANN) problems. Many hashing schemes aim at preserving the Euclidean distance of the original data. However, it is the geodesic distance rather than the Euclidean distance that more accurately characterizes the semantic similarity of data, especially in a high dimensional space. Consequently, manifold based hashing methods have achieved higher accuracy than conventional hashing schemes. To compute the geodesic distance, one should construct a nearest neighbor graph and invoke the shortest path algorithm, which is too expensive for a retrieval task. In this paper, we present a hashing scheme that preserves the geodesic distance and use a feasible out-of-sample method to generate the binary codes efficiently. The experiments show that our method outperforms several alternative hashing methods.
\end{abstract}

Keywords: Hashing, Manifold, Isomap, Out-of-sample extension

\section{Introduction}

Nearest Neighbor (NN) search is a basic and important step in image retrieval. For a large scale dataset of size $n$, the complexity of NN search is $O(n)$, which is still too high for big data processing. To solve this problem, sublinear approximate nearest neighbor methods have been proposed. Hashing methods are among such well-performed methods.

The basic idea of hashing is to use binary code to represent high-dimensional data, with similar data pair having smaller Hamming distance of binary codes. In other words, we should construct a mapping from high-dimension Euclidean space to Hamming space and preserve the original Euclidean distance of data in the Hamming space. We can see that hashing accelerates NN search on two aspects: one is that binary XOR operation to calculate the Hamming distance is much faster than calculating the Euclidean distance; the other is that binary code can be used as address to index the original data.

A simple and basic hash method is Locality-Sensitive Hashing(LSH) [2]. LSH is based on generating random hyperplane in Euclidean space. A hyperplane 
generates a bit of code. Data points on one side of the hyperplane are coded 0 at this bit, while at the other side are coded 1. Critically, points on the hyperplane can be coded either 0 or 1 , but the probability is very low. LSH successfully reduces the hashing process to sublinear query time with acceptable accuracy. However, because of its randomness, reaching higher accuracy needs longer code, and there is redundancy to some degree.

To get more compact binary code, some researchers started to analyze the inner structure of dataset. These efforts led to data-dependent hashing methods while LSH is data-independent method. Among the data-dependent methods, some are linear such as Iterative Quantization (ITQ) [7] and some are non-linear such as Spectral Hashing (SH) [14], and Anchor Graph Hashing (AGH) [9]. By learning the inner structure of dataset, the hash codes gain higher accuracy with shorter code length.

Although all the above hashing methods are concentrating on preserving Euclidean distance, however, the most similar data pair may not be the nearest neighbor in Euclidean space, but the nearest neighbor on the same manifold. Thus if we want to improve the precision of hashing method, we must take manifold structure into consideration as well. For linear manifold, linear methods such as PCAH, PCA-ITQ is applicable. But for non-linear manifold such as Swiss Roll, further exploration is needed to catch the manifold structure in hashing.

Graph methods play an important role in image analysis [15-17]. Many nonlinear manifold learning methods are motivated by graph approaches that create low-dimension embedding of data with manifold distribution in Euclidean space, such as Laplace Eigenmap (LE) [3], Locally Linear Embedding (LLE) [11], and Isometric Mapping (ISOMAP) [13]. Some manifold based hashing methods utilize these ideas and extend them to embedding in Hamming space, such as Inducitve Manifold Hashing (IMH) [12] and Locally Linear Hashing (LLH) [6]. IMH-tSNE extracts manifold structure with tSNE [1] and gives an out-of-sample extension scheme by locally linear property of manifold. LLH learns the locallylinear structure of manifold and preserves it in hash code.

In this paper, we utilize a widely used non-linear dimensionality reduction method Isometric Mapping (ISOMAP) to catch the geodesic distance structure of manifold and preserve it in hash code. We name the method Isometric Mapping Hashing (IsoMH). The experiments show that our IsoMH have comparable precision with the state-of-the-art methods.

\section{Isometric Mapping Hashing}

Given a set of data points $X=\left[\mathbf{x}_{1}, \mathbf{x}_{2}, \ldots, \mathbf{x}_{n}\right]^{T}\left(\mathbf{x}_{k} \in \mathbb{R}^{d}, X \in \mathbb{R}^{n \times d}\right)$, we want to get a mapping from $X$ to a binary hash code set $B \in\{-1,1\}^{n \times c}$ that the hamming distance in $B$ best approximates the data similarity in $X$.

Here, we explore the geodesic distance on manifold rather than the Euclidean distance as the quantitative measurement of data similarity. So in the process of mapping, we choose to preserve geodesic distance between data points in $X$ and 
then use a non-linear dimension reduction method ISOMAP (Section 2.1). After dimension reduction with ISOMAP, we got the low-dimensional embedding $Y \in \mathbb{R}^{n \times c}$, and we quantize $Y$ into a binary hash code $B$. Direct quantization with sign function $B=\operatorname{sign}(Y)$ may lead to great quantization loss. Therefore, we employ the iteration quantization method ITQ [7] to calculate $B$, which significantly reduces the quantization loss (Section 2.2). Finally we use an efficient out-of-sample extension method rather than doing ISOMAP again for a single data query (Section 3). The experiments to evaluate our method is introduced in Section 4.

Here is a list of mathematical symbols appearing in the following sections:

$b$ : number of bits of the final hash code.

$\xi$ : eigenvector of the kernel matrix.

$V$ : eigenvector matrix, $V=\left(\xi_{1}, \xi_{2}, \ldots, \xi_{b}\right)^{T}$.

$\lambda$ : eigenvalue of the kernel matrix

A: diagonal matrix of eigenvalues.

y: embedding vector.

$Y$ : data matrix, $Y=\left(\mathbf{y}_{1}, \mathbf{y}_{2}, \ldots, \mathbf{y}_{b}\right)^{T}$.

$B$ : binary code matrix.

\subsection{Dimensionality Reduction with ISOMAP}

Isomap is a non-linear dimensionality reduction method which aims at preserving the geodesic distance of the manifold structure. Real geodesic distance is difficult to compute, so Isomap takes shortest path distance to approximate the geodesic distance in large dataset.

With the dataset $X$, we can construct a neighborhood graph $G$, whose edges connect only neighboring nodes with the Euclidean distance of neighboring nodes as the weights. To properly define the neighborhood, we set a threshold $\epsilon$. Only node pairs whose Euclidean distances are below the threshold are considered as neighboring nodes. Alternately, we can choose a number $k$, only node pairs being k-nearest-neighbor to each other are regarded as neighboring. With this neighborhood graph $G$, we compute the shortest path distance between all node pairs in $G$ to approximate the geodesic distance of those node pairs. We denote the approximate geodesic distance matrix as $D$.

When using the Euclidean distance to reduce dimensionality, we come up with Multidimensional Scaling (MDS) [5]. MDS is a linear dimension reduction method aiming at preserving the pairwise distance. According to [5], we can get the low-dimensional embedding by decomposing the kernel matrix

$$
K=-\frac{1}{2} H D H
$$

where $H=E-\frac{1}{t} e e^{T}$. (What is $e$ ?) We pick eigenvectors $\xi_{k}(1 \leqslant k \leqslant b)$ of the top $b$ eigenvalues $\lambda_{k}(1 \leqslant k \leqslant b)$ of $K$. Then the low-dimensional embedding can be calculated as

$$
Y=\Lambda^{\frac{1}{2}} V
$$




\subsection{Quantize low-dimension embedding with ITQ}

After getting the low-dimension embedding $Y$, we are able to quantize it into binary code. In order to get the binary code, we need to quantize each dimension in the low-dimensional embedding into 0 and 1 according to the formula

$$
B=\operatorname{sign}(Y)
$$

Direct quantization may cause great loss as explained in [7]. So we try to calculate an optimized rotation matrix to minimize the quantization loss $Q$.

$$
Q=\|B-Y R\|_{F}
$$

Tackling this optimization problem, an iterative method was proposed to solve $B$ and $R$ one after the other [7]. When solving $B$, we fix $R$ and calculate $B$ with sign function $B=\operatorname{sign}(Y R)$. When solving $R$, we fix $B$ and the problem becomes the classic Orthogonal Procrustes Problem. It can be solved by computing the Singular Value Decomposition (SVD) of $B^{T} V$ as $S \Omega \hat{S}$, and we get $R=\hat{S} S^{T}$. Finally, we get a local minimum of the quantization loss $Q$ and its corresponding optimal rotation matrix $R$. Then binary hash code of training set can be represented as $B=\operatorname{sign}(Y R)$.

The whole process of training is described in pseudo-code in Algorithm 1.

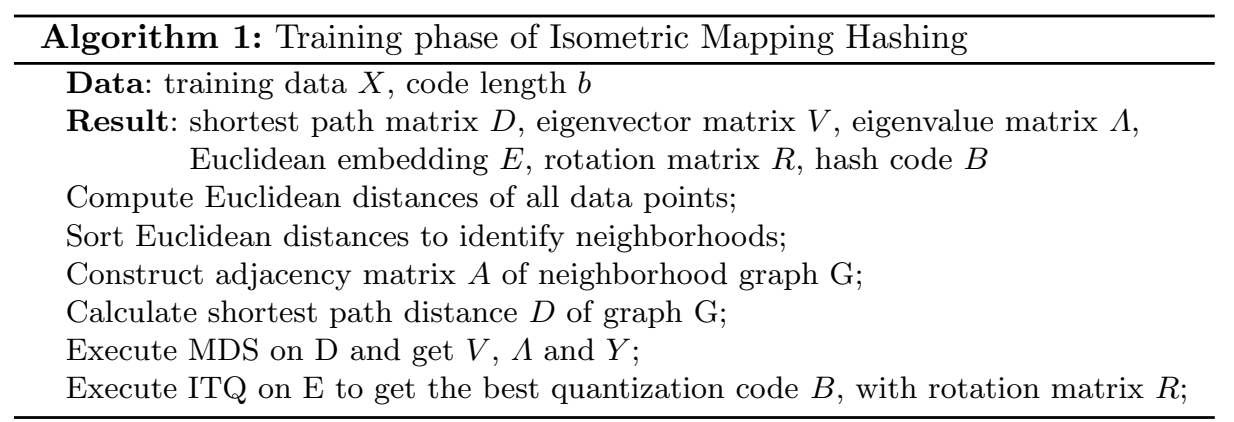

\section{Out-of-Sample Extension}

By applying the above described method on the training set, we can get the binary codes of the whole set. If we get a single testing data, we must calculate all other training data together with this single testing data. With the shortest path distance and matrix decomposition, the Isomap takes $O\left(n^{3}\right)$ to execute, which is difficult to meet the need of online query. To solve this problem, we adopt an outof-sample extension method [4] to calculate the approximate Isomap embedding.

Given a query $\mathbf{q}$, we first need to calculate its approximate geodesic distance to all other training set points, and that is, to calculate its shortest path distance 
to all others. In fact, there is no need to run Dijkstra one more time. If the neighborhood of $\mathbf{q}$ is $\mathbf{x}_{l_{1}}, \mathbf{x}_{l_{2}}, \ldots, \mathbf{x}_{l_{k}}\left(1 \leqslant l_{i} \leqslant n\right)$, then the shortest path distance of $\mathbf{q}$ to all other point in $X$ can be calculated by the shortest path distance of its neighbors. Denoting by $D\left(\mathbf{x}_{i}, \mathbf{x}_{j}\right)$, the shortest path distance from $\mathbf{x}_{i}$ to $\mathbf{x}_{j}$, it is obvious that

$$
D(\mathbf{x}, \mathbf{q})=\min _{i}\left\{\left\|\mathbf{q}-\mathbf{x}_{l_{i}}\right\|+D\left(\mathbf{x}_{l_{i}}, \mathbf{x}\right)\right\}
$$

To calculate a pair of shortest path distance, we just need to compare k-1 times and add $\mathrm{k}$ times.

With the newly calculated $D(\mathbf{x}, \mathbf{q})$, we can calculate the embedding $\mathbf{y}$ of an out-of-sample $\mathbf{x}$ according to the expression

$$
\mathbf{y}_{k}(\mathbf{q})=\frac{1}{2} \frac{1}{\sqrt{\lambda_{k}}} \sum_{i=1}^{n} v_{k i}\left(E_{\mathbf{x}^{\prime}}\left[D^{2}\left(\mathbf{x}^{\prime}, \mathbf{x}_{i}\right)\right]-D^{2}\left(\mathbf{x}_{i}, \mathbf{q}\right)\right)
$$

given in [4]. And it can be represented in matrix as

$$
\mathbf{y}=\frac{1}{2} \Lambda^{\frac{1}{2}} V \eta
$$

where $\eta=E_{\mathbf{x}^{\prime}}\left[D^{2}\left(\mathbf{x}^{\prime}, \cdot\right)\right]-D^{2}(\mathbf{q}, \cdot)$ is a column vector.

The pseudo-code of out-of-sample extension is written as follow.

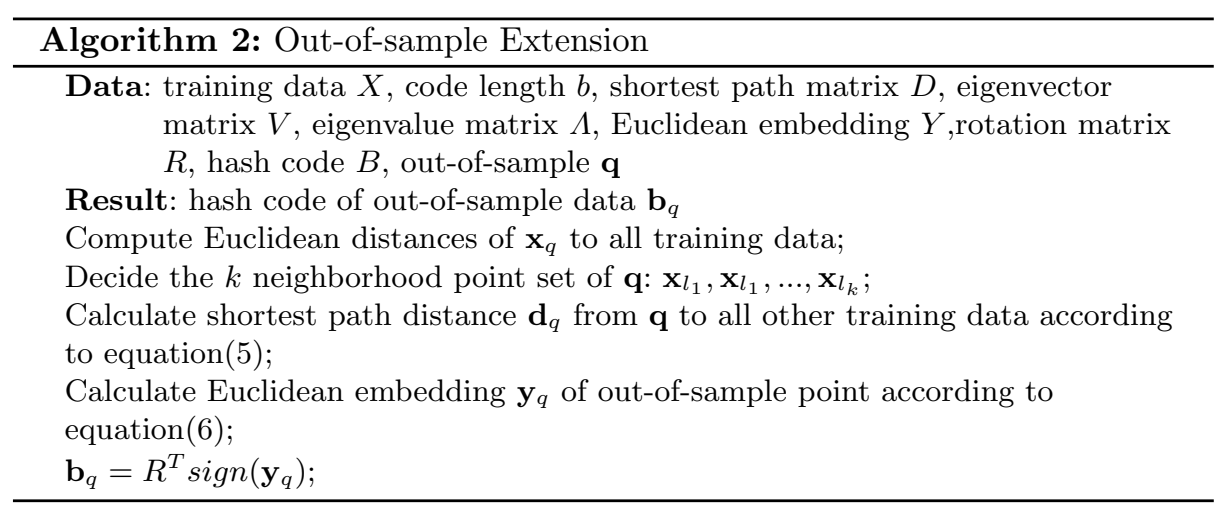

\section{Experiments}

We ran experiments to test our IsoMH method on two frequently-used image datasets: MNIST and CIFAR-10 [8]. 
MNIST. The MNIST dataset has $7000028 * 28$ small grayscale images of handwritten digits from ' 0 ' to ' 9 '. Each small image is represented by a 784dimensional vector and a single digit label. We used 2000 random images as the training set, and 500 random images as the query set.

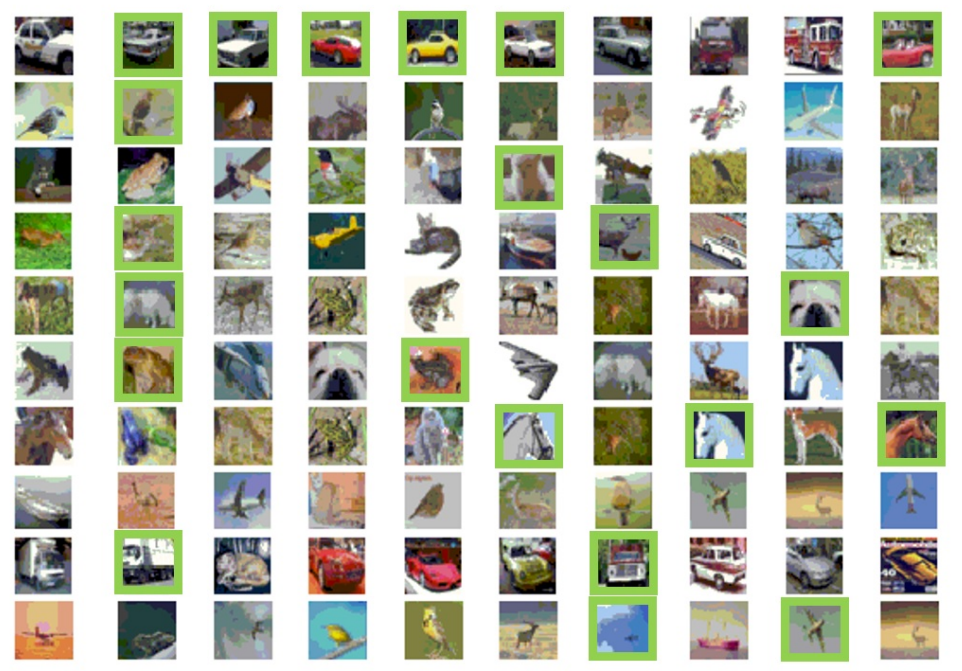

Fig. 1. Images in the left column are query images selected from 10 classes, and the rest images are the retrieval results using IsoMH. Images in green boxes represent the correct results.

CIFAR-10. There are $6000032 * 32$ small color images in CIFAR10. These small images are from 10 classes: airplane, automobile, bird, cat, deer, dog, frog, horse, ship, and truck. Each class has 6000 images. Every image in CIFAR10 is represented by a 320-dimensional GIST feature [10] vector and a label from ' 1 ' to ' 10 '. Fig. 1 shows sample results on CIFAR-10. We selected 10 images from different classes as the query and search the top 9 nearest neighbors in hamming distance of hash codes.

Besides IsoMH, we also tested other 5 unsupervised methods: LSH [2], PCAITQ [7], AGH [9], IMH-LE, and IMH-tSNE [1]. LSH and PCA-ITQ are linear while AGH, IMH-LE and IMH-tSNE are nonlinear. Specifically, two IMH methods are also manifold based. We used codes released by the original authors during the experiments.

Manifold based methods aim at getting semantically similar results. Though unsupervised, we used class labels as the ground truth, and evaluated the performance of those methods by Precision-Recall (P-R) curves and time consumption of training and query. We calculated the precision and recall by: 


$$
\begin{gathered}
\text { precision }=\frac{\text { Number of retrieved true neighbor pairs }}{\text { Number of retrieved neighbor pairs }} \\
\text { recall }=\frac{\text { Number of retrieved true neighbor pairs }}{\text { Total number of true neighbor pairs }}
\end{gathered}
$$

All our experiments were run on a 64-bit PC with $3.50 \mathrm{GHz}$ Intel i7-4770K CPU and 16.0GB RAM.

\subsection{Parameters Analysis}

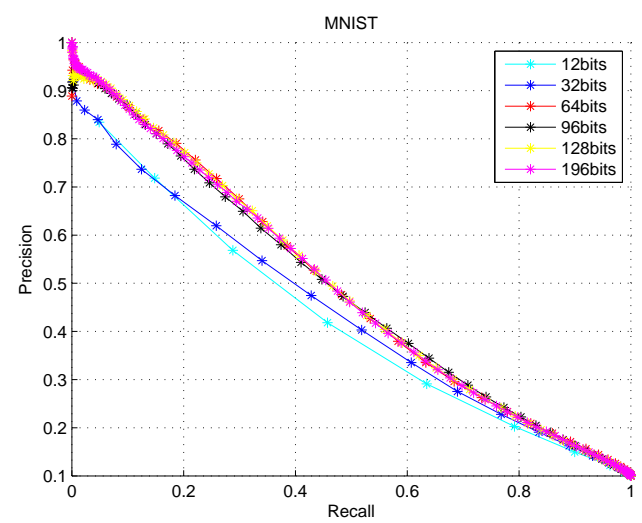

Fig. 2. The Precision-Recall curve of IsoMH with different hash code length on the MNIST dataset.

We set the hash code length to 12bits, 32bis, 64bits, 96bits and 128bits and performed the experiments, respectively. The results are shown in Fig 2. The curves show that when the number of bits is small, results will be better with the increasing of code length, and if the code length is large enough, adding hash bits cannot bring much advance in retrieval precision.

For the construction of nearest neighbor graph, different methods and parameters have almost the same results with shortest path distance algorithm, because the training set is large. In our experiments, we set $k=7$.

\subsection{Comparison to Other Methods}

Fig. 3 shows the P-R curves of the above-mentioned methods on MNIST. Our IsoMH performs better than all other methods. Linear method PCA-ITQ and manifold method IMH-tSNE are two most competitive methods. As the figure showing, IMH-tSNE performs better with longer code. For the training time 


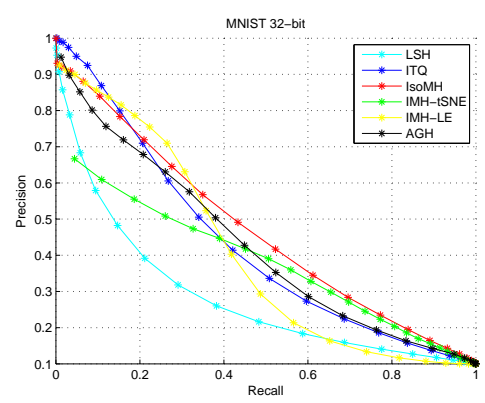

(a) 32-bits

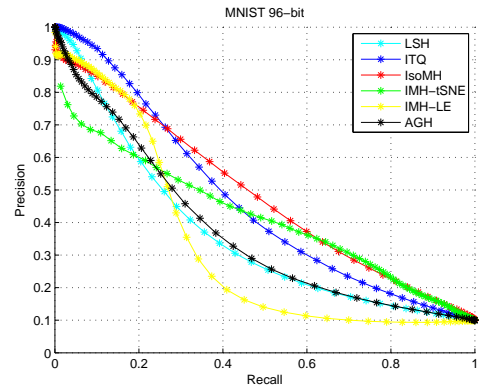

(c) 96-bits

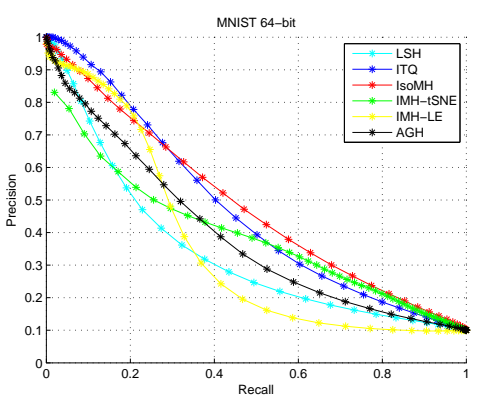

(b) 64-bits

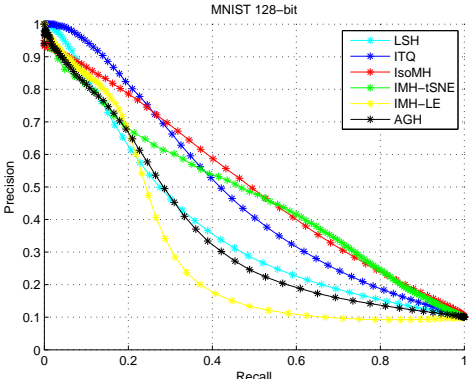

(d) 128-bits

Fig. 3. Precision-Recall curves of different methods with experiments on the MNIST dataset.

\begin{tabular}{|c|c|c|c|c|c|c|c|c|}
\hline \multirow{2}{*}{ Methods } & \multicolumn{2}{|c|}{32 bits } & \multicolumn{2}{c|}{64 bits } & \multicolumn{2}{c|}{96 bits } & \multicolumn{2}{c|}{128 bits } \\
\cline { 2 - 9 } & Training & Indexing & Training & Indexing & Training & Indexing & Training & Indexing \\
\hline IsoMH & 1853.44 & 189.03 & 1901.18 & 193.63 & 1954.06 & 211.14 & 2048.67 & 232.61 \\
PCA-ITQ & 10.61 & 0.18 & 18.27 & 0.35 & 29.78 & 0.50 & 42.52 & 0.69 \\
IMH-tSNE & 35.01 & 1.09 & 69.02 & 1.13 & 85.92 & 1.22 & 95.07 & 1.18 \\
IMH-LE & 96.38 & 2.20 & 90.61 & 2.20 & 87.13 & 2.52 & 87.99 & 2.28 \\
AGH & 84.02 & 10.92 & 87.42 & 13.35 & 84.97 & 13.20 & 85.47 & 13.09 \\
\hline
\end{tabular}

Table 1. Time consumption (milliseconds) of different methods on the MNIST dataset. 
consumption, as shown in Table 1, our method is not very competitive because the time complexity of shortest path algorithm is $O\left(n^{3}\right)$. In P-R curve, our IsoMH performs better in middle and lower precision region while be weaker in higher precision region.

Fig. 4 shows the P-R curves of results on CIFAR-10. CIFAR-10 has more complex images than MNIST so these P-R curves are lower than MNIST's. And our IsoMH is still better than all the curves.

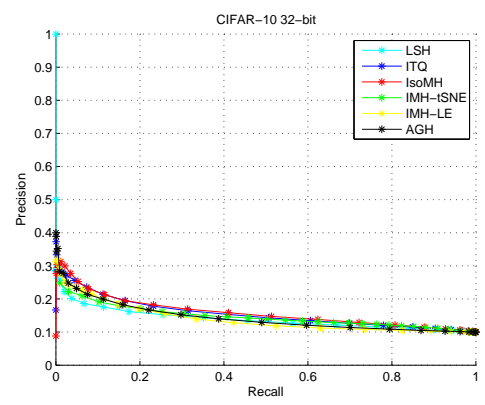

(a) 32-bits

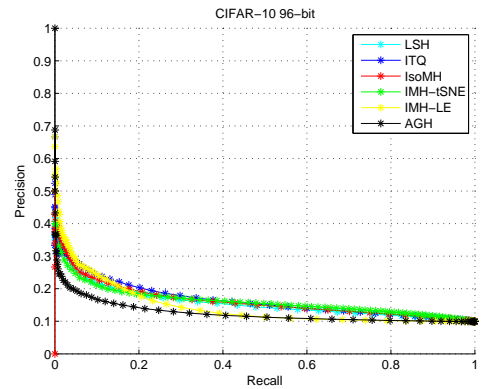

(c) 96-bits

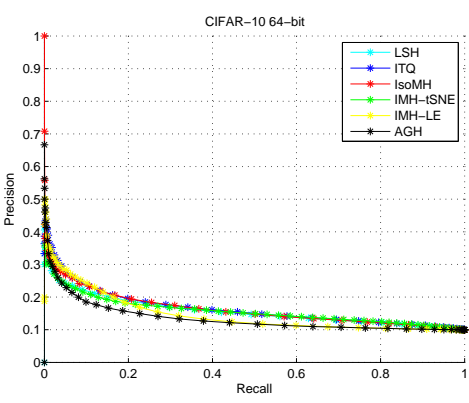

(b) 64-bits

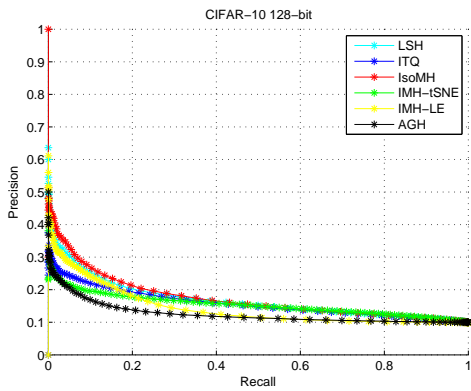

(d) 128-bits

Fig. 4. Precision-Recall curves of different methods with experiments on the CIFAR-10 dataset.

\section{Conclusion}

Manifold based hashing methods have advantages on retrieving semantically similar neighbors. In this paper, we preserve geodesic distance of manifold with binary code and utilize ISOMAP to implement our mapping. IsoMH outperforms many state-of-the-arts methods in precision but consume more time in computing the shortest distance of neighborhood graph. In the future work, we will concentrate on improving the time efficiency of IsoMH. 


\section{Acknowledgement}

This research is supported by National Natural Science Foundation of China (NSFC) projects No. 61370123 and No.61402389.

\section{References}

1. Visualizing data using t-SNE], author = Van der Maaten, Laurens and Hinton, Geoffrey, journal $=$ Journal of Machine Learning Research, year $=2008$, number $=2579-2605$, pages $=85$, volume $=9$

2. Andoni, A., Indyk, P.: Near-optimal hashing algorithms for approximate nearest neighbor in high dimensions. In: Foundations of Computer Science, 2006. FOCS'06. 47th Annual IEEE Symposium on. pp. 459-468. IEEE (2006)

3. Belkin, M., Niyogi, P.: Laplacian eigenmaps and spectral techniques for embedding and clustering. In: NIPS. vol. 14, pp. 585-591 (2001)

4. Bengio, Y., Paiement, J.f., Vincent, P., Delalleau, O., Roux, N.L., Ouimet, M.: Outof-sample extensions for LLE, Isomap, MDS, eigenmaps, and spectral clustering. In: Advances in Neural Information Processing Systems. pp. 177-184 (2004)

5. Cox, T.F., Cox, M.A.: Multidimensional Scaling. CRC Press (2010)

6. Go, I., Zhenguo, L., Xiao-Ming, W., Shih-Fu, C.: Locally linear hashing for extracting non-linear manifolds. In: Computer Vision and Pattern Recognition (CVPR), 2014 IEEE Conference on (2014)

7. Gong, Y., Lazebnik, S., Gordo, A., Perronnin, F.: Iterative quantization: A procrustean approach to learning binary codes for large-scale image retrieval. IEEE Transactions on Pattern Analysis and Machine Intelligence 35(12), 2916-2929 (2013)

8. Krizhevsky, A., Hinton, G.: Learning multiple layers of features from tiny images. Master's thesis, Department of Computer Science, University of Toronto (2009)

9. Liu, W., Wang, J., Kumar, S., Chang, S.F.: Hashing with graphs. In: Proceedings of the 28th International Conference on Machine Learning (ICML-11). pp. 1-8 (2011)

10. Oliva, A., Torralba, A.: Modeling the shape of the scene: A holistic representation of the spatial envelope. International journal of computer vision $42(3), 145-175$ (2001)

11. Roweis, S.T., Saul, L.K.: Nonlinear dimensionality reduction by locally linear embedding. Science 290(5500), 2323-2326 (2000)

12. Shen, F., Shen, C., Shi, Q., Van Den Hengel, A., Tang, Z.: Inductive hashing on manifolds. In: Computer Vision and Pattern Recognition (CVPR), 2013 IEEE Conference on. pp. 1562-1569. IEEE (2013)

13. Tenenbaum, J.B., De Silva, V., Langford, J.C.: A global geometric framework for nonlinear dimensionality reduction. Science 290(5500), 2319-2323 (2000)

14. Weiss, Y., Torralba, A., Fergus, R.: Spectral hashing. In: Advances in neural information processing systems. pp. 1753-1760 (2009)

15. Xiao, B., Hancock, E.R., Wilson, R.C.: Graph characteristics from the heat kernel trace. Pattern Recognition 42(11), 2589-2606 (2009)

16. Xiao, B., Hancock, E.R., Wilson, R.C.: Geometric characterization and clustering of graphs using heat kernel embeddings. Image and Vision Computing 28(6), 1003$1021(2010)$ 
17. Zhang, H., Bai, X., Zhou, J., Cheng, J., Zhao, H.: Object detection via structural feature selection and shape model. IEEE transactions on image processing 22(1112), 4984-4995 (2013) 